\title{
О ПРЕДЕЛАХ ВЕРШИННО-СИММЕТРИЧЕСКИХ ГРАФОВ И ИХ АВТОМОРФИЗМАХ
}

\section{В. И. Трофимов}

\begin{abstract}
С использованием простого, но весьма общего способа построения графов Кэли с тривиальным стабилизатором вершины строится пример бесконечного локально конечного графа Кэли (и, следовательно, пример бесконечного связного локально конечного вершинно-симметрического унимодулярного графа), изолированного в пространстве связных локально конечных вершинно-симметрических графов. Приводятся также примеры неизолированных в этом пространстве графов Кэли, которые изолированы от множества связных вершинно-симметрических конечных графов.

Ключевые слова: связный локально конечный вершинно-симметрический граф, граф Кэли, сходимость графов.

V. I. Trofimov. On limits of vertex-symmetric graphs and their automorphisms.

Using a simple but rather general method of constructing Cayley graphs with trivial vertex stabilizers, we give an example of an infinite locally finite Cayley graph (and, hence, an example of an infinite connected locally finite vertex-symmetric unimodular graph) which is isolated in the space of connected locally finite vertex-symmetric graphs. We also give examples of Cayley graphs which are not isolated in this space but are isolated from the set of connected vertex-symmetric finite graphs.
\end{abstract}

Keywords: connected locally finite vertex-symmetric graph, Cayley graph, convergence of graphs.

MSC: 05C25, 20F65, 20F69

DOI: $10.21538 / 0134-4889-2019-25-4-226-234$

\section{1. Введение и вспомогательные результаты}

Настоящая заметка возникла в связи с [8]. В ней показывается, что простые наблюдения, касающиеся сходимости связных локально конечных вершинно-симметрических графов, в сочетании с одним способом построения графов Кэли с тривиальным стабилизатором вершины, а также некоторыми известными результатами делают возможным указать в пространстве связных локально конечных вершинно-симметрических графов изолированный бесконечный граф Кэли (и, следовательно, изолированный бесконечный унимодулярный граф, что отвечает на вопрос из [8]) и неизолированный граф Кэли, который изолирован от множества связных вершинно-симметрических конечных графов.

Под графом в этой заметке понимается неориентированный граф без петель и без кратных ребер. Если $\Gamma$ - граф, то $V(\Gamma)$ - множество его вершин, $E(\Gamma)$ - множество его ребер, $d_{\Gamma}(.,$.$) - обычное расстояние (метрика, если \Gamma$ связен) на $V(\Gamma)$, Aut $(\Gamma)$ - группа автоморфизмов графа $\Gamma$ (рассматриваемых как подстановки на $V(\Gamma)$ ). Далее, если $x \in V(\Gamma)$, то $\Gamma(x)=\{y \in V(\Gamma):\{x, y\} \in E(\Gamma)\}-$ окрестность $x$ в $\Gamma, B_{\Gamma}(x, r)=\left\{y \in V(\Gamma): d_{\Gamma}(x, y) \leq r\right\}$, где $r \in \mathbb{R}$, - шар радиуса $r$ с центром $x$ графа $\Gamma, G_{x}$, где $G \leq \operatorname{Aut}(\Gamma),-$ стабилизатор вершины $x$ в группе $G$. Кроме того, $\langle X\rangle_{\Gamma}$, где $X \subseteq V(\Gamma)$, - подграф графа $\Gamma$, порожденный $X$. Граф $\Gamma$ вершинно-симметричен, если $\operatorname{Aut}(\Gamma)$ транзитивна на $V(\Gamma)$. Для группы $G$ и системы ее порождающих $M=M^{-1} \not \supset 1$ через $\Gamma_{G, M}$ обозначается граф Кэли группы $G$, построенный по $M$.

Пусть $\mathfrak{G}$ - множество всех (классов изоморфных) связных локально конечных вершинносимметрических графов, наделенное следующей метрикой $\rho(.,):$. для $\Gamma_{1}, \Gamma_{2} \in \mathfrak{G}, \Gamma_{1} \nsucceq \Gamma_{2}$, 
$\rho\left(\Gamma_{1}, \Gamma_{2}\right)=2^{-n}$, где $n=\max \left\{m \in \mathbb{N} \cup\{0\}:\left\langle B_{\Gamma_{1}}\left(x_{1}, m\right)\right\rangle_{\Gamma_{1}} \cong\left\langle B_{\Gamma_{2}}\left(x_{2}, m\right)\right\rangle_{\Gamma_{2}}, x_{1} \in V\left(\Gamma_{1}\right), x_{2} \in\right.$ $\left.V\left(\Gamma_{2}\right)\right\}$ (здесь и далее $\mathbb{N}-$ множество целых положительных чисел). Во многом обязанное своим появлением работам [9] и [1] метрическое пространство связных локально конечных вершинно-симметрических графов $\mathfrak{G}$ существенно используется в [2;3]. Далее в этом разделе при описании простых свойств пространства $\mathfrak{G}$ мы во многом следуем $[2, \text { п. } 1]^{1}$ и $[3, \S 2]$.

Нам будет удобно зафиксировать следующую ситуацию, возникающую при сходимости в $\mathfrak{G}$ последовательности $\left(\Gamma_{i}\right)_{i \in \mathbb{N}} \mathrm{K} \Gamma$.

$\left(^{*}\right)\left(\Gamma_{i}\right)_{i \in \mathbb{N}}-$ последовательность графов из $\mathfrak{G}$, сходящаяся к $\Gamma \in \mathfrak{G}, x \in V(\Gamma), x_{i} \in V\left(\Gamma_{i}\right)$ для $i \in \mathbb{N}, \varphi_{i}$ для $i \in \mathbb{N}$ - изоморфизм $\left\langle B_{\Gamma}\left(x, n_{i}\right)\right\rangle_{\Gamma}$ на $\left\langle B_{\Gamma_{i}}\left(x_{i}, n_{i}\right)\right\rangle_{\Gamma_{i}}$, отображающий $x$ в $x_{i}$, где $n_{i} \in \mathbb{N}$ и $n_{i} \rightarrow \infty$ при $i \rightarrow \infty$.

Предположим, что имеет место $(*)$. Тогда, если $y_{1}, \ldots, y_{k}, z_{1}, \ldots, z_{k}-$ вершины графа $\Gamma$ такие, что для бесконечного множества значений $i$ существует автоморфизм $g_{i}$ графа $\Gamma_{i}$, отображающий $\varphi_{i}\left(y_{j}\right)$ в $\varphi_{i}\left(z_{j}\right)$ для всех $1 \leq j \leq k$, то существует такой автоморфизм $g$ графа $\Gamma$, что $g\left(y_{j}\right)=z_{j}$ для всех $1 \leq j \leq k$. Отсюда следует, что для произвольного фиксированного $r \in \mathbb{N}$ для всех достаточно больших $i$ группа $\varphi_{i}^{-1} \operatorname{Aut}\left(\Gamma_{i}\right)_{x_{i}} \varphi_{i}$ идуцирует на $B_{\Gamma}(x, r)$ подгруппу группы, индуцируемой $\operatorname{Aut}(\Gamma)_{x}$ на $B_{\Gamma}(x, r)$. В частности, если для некоторого $r \in \mathbb{N}$ поэлементный стабилизатор $B_{\Gamma}(x, r-1)$ в $\operatorname{Aut}(\Gamma)_{x}$ действует тривиально на $B_{\Gamma}(x, r)$, то для всех достаточно больших $i$ поэлементный стабилизатор $B_{\Gamma_{i}}\left(x_{i}, r-1\right)$ в $\operatorname{Aut}\left(\Gamma_{i}\right)_{x_{i}}$ действует тривиально на $B_{\Gamma_{i}}\left(x_{i}, r\right)$. Поскольку (в силу вершинной транзитивности $\operatorname{Aut}(\Gamma)$ и $\left.\operatorname{Aut}\left(\Gamma_{i}\right)\right)$ последние условия эквивалентны соответственно точности действия $\operatorname{Aut}(\Gamma)_{x}$ на $B_{\Gamma}(x, r-1)$ и точности действия $\operatorname{Aut}\left(\Gamma_{i}\right)_{x_{i}}$ на $B_{\Gamma_{i}}\left(x_{i}, r-1\right)$, то справедливо следующее утверждение.

Предложение 1. Если Aut $(\Gamma)_{x}$ - конечная группа, то для всех достаточно больших $i$ имеем $\operatorname{Aut}\left(\Gamma_{i}\right)_{x_{i}} \lesssim \operatorname{Aut}(\Gamma)_{x}$. В частности, если $\operatorname{Aut}(\Gamma)_{x}=1$, mо $\operatorname{Aut}\left(\Gamma_{i}\right)_{x_{i}}=1$ для всех достаточно больиих $i$.

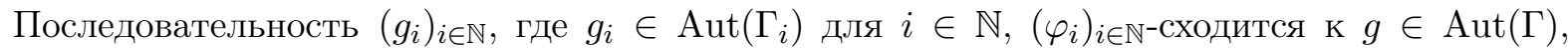
если для произвольной вершины $y$ графа $\Gamma$ и всех достаточно больших $i$ (вершины $\varphi_{i}(y)$ и $\varphi_{i}(g(y))$ определены и) $g_{i}\left(\varphi_{i}(y)\right)=\varphi_{i}(g(y))$ (см. [3, §2]). Далее, пусть $G_{i} \leq \operatorname{Aut}\left(\Gamma_{i}\right)$ для всех $i \in \mathbb{N}$. Тогда автоморфизм $g$ графа $\Gamma$ называется $\left(\varphi_{i}\right)_{i \in \mathbb{N}}$-предельным для $\left(G_{i}\right)_{i \in \mathbb{N}}$, если для некоторой возрастающей последовательности натуральных чисел $\left(i_{t}\right)_{t \in \mathbb{N}}$ найдутся такие

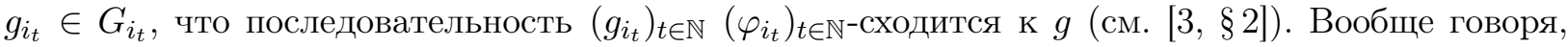
множество $\left(\varphi_{i}\right)_{i \in \mathbb{N}}$-предельных для $\left(G_{i}\right)_{i \in \mathbb{N}}$ автоморфизмов графа $\Gamma$ не является подгруппой группы $\operatorname{Aut}(\Gamma)$. Однако, как легко заметить, справедливо следующее утверждение.

Предложение 2. Предположим, что имеет место $(*) u G_{i} \leq \operatorname{Aut}\left(\Gamma_{i}\right), i \in \mathbb{N},-$ вершиннотранзитивные группы. Тогда группа $\operatorname{Aut}(\Gamma)$ содержит вершинно-транзитивную подгруппу, состояшую из $\left(\varphi_{i}\right)_{i \in \mathbb{N}}$-предельных для $\left(G_{i}\right)_{i \in \mathbb{N}}$ автоморфизмов графа $Г$. Более того, существуют такая возрастающая последовательности натуральных чисел $\left(i_{t}\right)_{t \in \mathbb{N}}$ и такая вершиннотранзитивная подгруппа $G$ группы Aut $(\Gamma)$, что для каждого $g \in G$ найдутся $g_{i_{t}} \in G_{i_{t}}, t \in \mathbb{N}$, для которых последовательность $\left(g_{i_{t}}\right)_{t \in \mathbb{N}}\left(\varphi_{i_{t}}\right)_{t \in \mathbb{N}}$-сходится $\kappa g$.

В качестве следствия получаем

Предложение 3. Если $\left(\Gamma_{i}\right)_{i \in \mathbb{N}}-$ последовательность графов Кэли из $\mathfrak{G}$, сходлщаяся к $\Gamma \in \mathfrak{G}$, то Г такэе является графом Кэли. Другими словами, в $\mathfrak{G}$ графы Кэли групп образуют замкнутое подмножество.

Д о к а з а т е л ь с т в о. Будем предполагать (без потери общности), что для $\left(\Gamma_{i}\right)_{i \in \mathbb{N}}$ и $Г$ выполняется $(*)$. Тогда, если $\Gamma_{i}$ есть граф Кэли группы $G_{i}$, то Г есть граф Кэли группы $G$, для

\footnotetext{
${ }^{1}$ Пользуясь случаем, укажем на имеющиеся в [2] опечатки: на с. 150 в строке 14 вместо "изоморфно" должно быть "содержит подгруппу, изоморфную", а в строке 23 вместо $\cong$ должно быть .
} 
которой имеется последовательность натуральных чисел $\left(i_{t}\right)_{t \in \mathbb{N}}$ с указанным в предложении 2 свойством.

Формулировке предложения 4 предпошлем определения (см. [7]). Подмножество группы называется дискриминирующим, если оно не содержит 1 и имеет непустое пересечение с каждой неединичной нормальной подгруппой группы. Группа, обладающая конечным дискриминирующим подмножеством, называется конечно дискриминируемой. По [7, Proposition 2.2] группа изолирована тогда и только тогда, когда она конечно определена и обладает конечным дискриминирующим подмножеством. (Определение изолированной группы см. в [7]; однако в дальнейшем нами используется, по существу, лишь указанная характеризация таких групп.)

Предложение 4. Пусть $G$ - изолированная группа, порожденная конечным множеством $M=M^{-1} \not \supset 1$. Предположим, что стабилизатор вершины графа $\Gamma_{G, M}$ в группе $\operatorname{Aut}\left(\Gamma_{G, M}\right)$ тривиален. Тогда граф $\Gamma_{G, M}$ изолирован в $\mathfrak{G}$.

Д о к а з а т е л ь с т в о. Предположим, что, напротив, имеется последовательность $\left(\Gamma_{i}\right)_{i \in \mathbb{N}}$ связных вершинно-симметрических графов, сходящаяся к $\Gamma:=\Gamma_{G, M}$, причем $\Gamma_{i} \nsucceq \Gamma$ для всех $i \in \mathbb{N}$. Далее мы используем применительно к $\left(\Gamma_{i}\right)_{i \in \mathbb{N}}$ и $\Gamma$ обозначения из $(*)$, причем предполагаем, что $x$ есть вершина 1 графа Кэли $\Gamma_{G, M}$ (и следовательно, окрестность $x$ в графе Г есть $M)$.

Поскольку $\operatorname{Aut}(\Gamma)_{x}=1$, то согласно предложению 1 для всех достаточно больших $i$, скажем, для всех $i>i_{0}$, где $i_{0} \in \mathbb{N}$, имеем $\operatorname{Aut}\left(\Gamma_{i}\right)_{x_{i}}=1$ и, следовательно, $\Gamma_{i}$ является графом Кэли группы $G_{i}:=\operatorname{Aut}\left(\Gamma_{i}\right)$, построенным по некоторой системе ее порождающих $M_{i}=M_{i}^{-1} \not \supset 1$. Для таких $i$ будем, не теряя общности, считать, что $x_{i}$ есть вершина 1 графа Кэли $\Gamma_{G_{i}, M_{i}}$ (и следовательно, окрестность $x_{i}$ в графе $\Gamma_{i}$ есть $\left.M_{i}\right)$. Из $\operatorname{Aut}(\Gamma)_{x}=1$ следует, кроме того, что $\operatorname{Aut}(\Gamma)$ совпадает с группой левых сдвигов $G$ и является единственной вершинно-транзитивной группой автоморфизмов графа Г. Согласно предложению 2 это влечет существование такой возрастающей последовательности натуральных чисел $\left(i_{t}\right)_{t \in \mathbb{N}}$, где $i_{1}>i_{0}$, что для каждого $g \in G$ найдутся $g_{i_{t}} \in G_{i_{t}}, t \in \mathbb{N}$, для которых последовательность $\left(g_{i_{t}}\right)_{t \in \mathbb{N}}\left(\varphi_{i_{t}}\right)_{t \in \mathbb{N}}$-сходится к $g$.

Пусть $M=\left\{h_{1}, \ldots, h_{d}\right\}$. Для каждого $1 \leq s \leq d$ пусть $h_{s, t} \in G_{i_{t}}, t \in \mathbb{N}$, таковы, что последо-

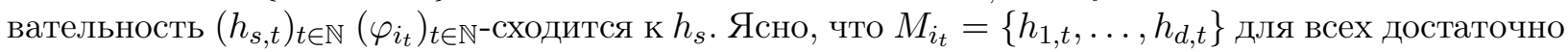
больших $t$. Далее, для произвольного произведения $h_{j_{1}} \ldots h_{j_{k}}$, где $j_{1}, \ldots, j_{k} \in\{1, \ldots, d\}$, после-

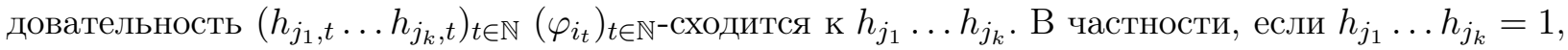
то для всех достаточно больших $t$ имеем $h_{j_{1}, t} \ldots h_{j_{k}, t}\left(x_{i_{t}}\right)=x_{i_{t}}$, что с учетом тривиальности стабилизатора $x_{i_{t}}$ в $G_{i_{t}}$ для всех $t \in \mathbb{N}$ влечет $h_{j_{1}, t} \ldots h_{j_{k}, t}=1$ для всех достаточно больших $t$. В силу конечной определенности изолированной группы $G$ отсюда следует, что для всех достаточно больших $t$ отображение $h_{s} \mapsto h_{s, t}, 1 \leq s \leq d$, продолжается до гомоморфизма группы $G$ на группу $G_{i_{t}}$. Предположим, что для бесконечного множества значений $t \in \mathbb{N}$ эти гомоморфизмы определены и имеют нетривиальное ядро. Тогда, поскольку изолированная группа $G$ имеет конечное дискриминирующее подмножество, найдется произведение $h_{j_{1}} \ldots h_{j_{k}}$, где $j_{1}, \ldots, j_{k} \in\{1, \ldots, d\}$, принадлежащее этим ядрам для бесконечного множества значений $t \in \mathbb{N}$, но отличное от 1 . Для бесконечного множества значений $t \in \mathbb{N}$ имеем, следова-

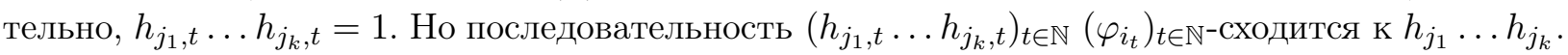
Поэтому $h_{j_{1}} \ldots h_{j_{k}}(x)=x$, что влечет $h_{j_{1}} \ldots h_{j_{k}}=1$, а это противоречит выбору $h_{j_{1}} \ldots h_{j_{k}}$. Таким образом, для всех достаточно больших $t$ имеется изоморфизм группы $G$ на группу $G_{i_{t}}$, отображающий $M$ на $M_{i_{t}}$, а потому и изоморфизм графа $\Gamma=\Gamma_{G, M}$ на граф $\Gamma_{i_{t}}=\Gamma_{G_{i_{t}}, M_{i_{t}}}$. Полученное противоречие завершает доказательство предложения 4.

В заключение этого раздела сделаем несколько замечаний относительно модулярных функций для графов и свойства унимодулярности (ср. [4]). Пусть $\Gamma \in \mathfrak{G}$. Тогда группа Aut(Г), наделенная естественной топологией поточечной сходимости, хаусдорфова и локально компактна. В частности, для произвольной замкнутой подгруппы $H$ группы $\operatorname{Aut}(\Gamma)$ на $\sigma$-кольце, порожденном всеми компактными подмножествами группы $H$, определена (однозначно с точностью до вещественного положительного постоянного множителя) левоинвариантная мера 
Хаара $\mu_{H}$, а на $H$ определена модулярная функция $\Delta_{H}$ (являющаяся гомоморфизмом группы $H$ в мультипликативную группу положительных вещественных чисел), причем для произвольных $x \in V(\Gamma)$ и $h \in H$ имеем $0<\mu_{H}\left(H_{x}\right)<\infty$ и

$$
\begin{gathered}
\Delta_{H}(h)=\mu_{H}\left(H_{h^{-1}(x)}\right) / \mu_{H}\left(H_{x}\right)=\left|H_{h^{-1}(x)}: H_{h^{-1}(x)} \cap H_{x}\right| /\left|H_{x}: H_{h^{-1}(x)} \cap H_{x}\right| \\
=\left|H_{h^{-1}(x)}(x)\right| /\left|H_{x}\left(h^{-1}(x)\right)\right|=\left|H_{x}(h(x))\right| /\left|H_{x}\left(h^{-1}(x)\right)\right| .
\end{gathered}
$$

Замкнутая подгруппа $H$ группы $\operatorname{Aut}(\Gamma)$ называется унимодулярной, если $\Delta_{H}$ всюду на $H$ принимает единичное значение; граф $\Gamma$ называется унимодулярным, если группа Aut(Г) унимодулярна. Если $H$ - вершинно-транзитивная замкнутая группа автоморфизмов графа $Г$ и $x \in V(\Gamma)$, то $H$ порождается компактной подгруппой $H_{x}$ (на которой $\Delta_{H}$ всюду принимает единичное значение) и произвольным набором элементов $\left\{h_{i}: i \in I\right\}$ группы $H$ таким, что $\Gamma(x)=\left\{h_{i}(x): i \in I\right\}$. Следовательно, в этом случае унимодулярность $H$ эквивалентна выполнению равенства $\Delta_{H}\left(h_{i}\right)=1$ для всех $i \in I$, что с учетом (1) эквивалентно, в свою очередь, совпадению для каждой $H_{x}$-орбиты на $\Gamma(x)$ ее длины с длиной спаренной с ней (в группе $H$ ) $H_{x}$-орбиты на $\Gamma(x)$. (Напомним, что если $H$ - произвольная вершинно-транзитивная группа

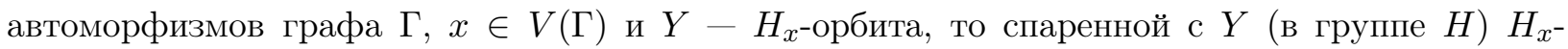
орбитой называется $H_{x}$-орбита $Y^{*}=\left\{h^{-1}(x): h \in H\right.$ и $\left.h(x) \in Y\right\}$.) Замечая, кроме того, что для произвольной вершинно-транзитивной группы $H$ автоморфизмов графа $\Gamma$ и $x \in V(\Gamma)$ стабилизатор $x$ в замыкании $H$ в группе $\operatorname{Aut}(\Gamma)$ имеет на $\Gamma(x)$ те же орбиты, что $H_{x}$, получаем справедливость утверждения (1) следующего предложения.

Предложение 5. (1) Пусть $\Gamma \in \mathfrak{G} u x \in V(\Gamma)$. Если $H$ - вериинно-транзитивная группа автоморфизмов графа $\Gamma$, то ее замыкание в $\operatorname{Aut}(\Gamma)$ тогда и только тогда является унимодулярной группой, когда длина каждой $H_{x}$-орбиты на $\Gamma(x)$ совпадает с длиной спаренной с ней (в группе $H) H_{x}$-орбиты на $\Gamma(x)$.

(2) Если $\Gamma \in \mathfrak{G}$ и $H \leq G-$ вершинно-транзитивные замкнутые группы автоморфизмов графа $\Gamma$, то из унимодулярности $H$ следует унимодулярность $G$. В частности, если $\Gamma \in \mathfrak{G}$ допускает вершинно-транзитивную замкнутую унимодулярную группу автоморфизмов, то Г унимодулярен.

Д о к а з а т е л ь с т в о. Утверждение (1) предложения доказано выше. Докажем утверждение (2). Пусть $\Gamma \in \mathfrak{G}$ и $H \leq G$ - вершинно-транзитивные замкнутые группы автоморфизмов графа Г, причем $H$ унимодулярна. Согласно (1) для $x \in V(\Gamma)$ длина каждой $H_{x}$-орбиты на $\Gamma(x)$ совпадает с длиной спаренной с ней (в группе $H$ ) $H_{x}$-орбиты на $\Gamma(x)$. Но каждая $G_{x}$-орбита на $\Gamma(x)$ есть объединение некоторого набора $H_{x}$-орбит на $\Gamma(x)$, причем спаренная с ней (в группе $G$ ) $G_{x}$-орбита на $\Gamma(x)$ есть объединение $H_{x}$-орбит на $\Gamma(x)$, спаренных с $H_{x \text {-ор- }}$ битами из этого набора (в группе $H$ ). Поэтому длина каждой $G_{x}$-орбиты на $\Gamma(x)$ совпадает с длиной спаренной с ней (в группе $G$ ) $G_{x}$-орбиты на $\Gamma(x)$, что согласно (1) влечет унимодулярность группы $G$. Предложение доказано.

3 а м е ч а н и е 1. Вершинно-транзитивная замкнутая группа автоморфизмов унимодулярного графа $\Gamma \in \mathfrak{G}$ (например, регулярного дерева валентности 3 ) может не быть унимодулярной.

Следствием предложения 5 является унимодулярность графа $\Gamma \in \mathfrak{G}$, являющегося графом Кэли или, более общо, допускающего вершинно-транзитивную дискретную группу автоморфизмов.

\section{2. Способ получения графов Кэли с тривиальным стабилизатором вершины}

Предложение 7 настоящего раздела дает весьма общий способ построения графов Кэли с тривиальным стабилизатором вершины в группе всех автоморфизмов графа. Предложение 6 используется при доказательстве предложения 7. 
Предложение 6. Пусть $F-$ свободная группа со свободными порождающими $x_{1}, x_{2}, u$ nycms

$$
D:=\left\{x_{1}, x_{1}^{-1}, x_{2}, x_{2}^{-1}, x_{1}^{2}, x_{1}^{-2}, x_{1} x_{2}, x_{2}^{-1} x_{1}^{-1}, x_{2}^{4}, x_{2}^{-4}\right\} .
$$

Обозначим через $\Delta$ подграф графа $\Gamma_{F, D}$, порожденный шаром радиуса 2 с иентром в 1 гра$\oint a \Gamma_{F, D}$. Тогда автоморфизмы графа $\Delta$, стабилизирующие вершину 1 , поэлементно стабилизируют множество $\Delta(1)=D$.

Д о к а з а т е л ь с т в о. Хотя здесь можно было ограничиться указанием, что справедливость утверждения предложения несложно устанавливается непосредственной проверкой, мы приведем соответствующие аргументы.

Смежными с вершиной 1 в графе $\Delta$ являются следующие вершины:

$x_{1}$, для которой $\Delta\left(x_{1}\right) \cap \Delta(1)=\left\{x_{1}^{-1}, x_{1}^{2}, x_{1} x_{2}\right\}$;

$x_{1}^{-1}$, для которой $\Delta\left(x_{1}^{-1}\right) \cap \Delta(1)=\left\{x_{1}, x_{2}, x_{1}^{-2}\right\}$;

$x_{2}$, для которой $\Delta\left(x_{2}\right) \cap \Delta(1)=\left\{x_{1}^{-1}\right\}$;

$x_{2}^{-1}$, для которой $\Delta\left(x_{2}^{-1}\right) \cap \Delta(1)=\left\{x_{2}^{-1} x_{1}^{-1}\right\}$;

$x_{1}^{2}$, для которой $\Delta\left(x_{1}^{2}\right) \cap \Delta(1)=\left\{x_{1}\right\}$;

$x_{1}^{-2}$, для которой $\Delta\left(x_{1}^{-2}\right) \cap \Delta(1)=\left\{x_{1}^{-1}\right\}$;

$x_{1} x_{2}$, для которой $\Delta\left(x_{1} x_{2}\right) \cap \Delta(1)=\left\{x_{1}\right\}$;

$x_{2}^{-1} x_{1}^{-1}$, для которой $\Delta\left(x_{2}^{-1} x_{1}^{-1}\right) \cap \Delta(1)=\left\{x_{2}^{-1}\right\}$;

$x_{2}^{4}$, для которой $\Delta\left(x_{2}^{4}\right) \cap \Delta(1)=\varnothing$;

$x_{2}^{-4}$, для которой $\Delta\left(x_{2}^{-4}\right) \cap \Delta(1)=\varnothing$.

Отсюда следует, что каждый автоморфизм графа $\Delta$, стабилизирующий вершину 1 , оставляет на месте следующие множества: $\left\{x_{1}, x_{1}^{-1}\right\},\left\{x_{2}, x_{1}^{2}, x_{1}^{-2}, x_{1} x_{2}\right\}\left(=\left\{\left(\left(\Delta\left(x_{1}\right) \cap \Delta(1)\right) \cup\left(\Delta\left(x_{1}^{-1}\right) \cap\right.\right.\right.\right.$ $\left.\Delta(1))) \backslash\left\{x_{1}, x_{1}^{-1}\right\}\right),\left\{x_{2}^{4}, x_{2}^{-4}\right\},\left\{x_{2}^{-1}, x_{2}^{-1} x_{1}^{-1}\right\}$.

Предположим, что некоторый автоморфизм $a$ графа $\Delta$ стабилизирует вершину 1 , но меняем местами вершины $x_{1}$ и $x_{1}^{-1}$. Тогда а также меняет местами множества $\Delta\left(x_{1}\right) \cap \Delta(1)$ и $\Delta\left(x_{1}^{-1}\right) \cap \Delta(1)$ и потому меняет местами множества $\left\{x_{1}^{2}, x_{1} x_{2}\right\}$ и $\left\{x_{2}, x_{1}^{-2}\right\}$. В частности, $a\left(x_{2}\right) \in\left\{x_{1}^{2}, x_{1} x_{2}\right\}$. Но в графе $\Delta$ имеется не проходящий через 1 путь длины 3 , начинающийся в $x_{2}$ и заканчивающийся в $x_{2}^{4}$, и в то же время отсутствует не проходящий через 1 путь длины 3 , начинающийся в одной из вершин $x_{1}^{2}, x_{1} x_{2}$ и заканчивающийся в одной из вершин $x_{2}^{4}, x_{2}^{-4}$, что противоречит $a$-допустимости множества $\left\{x_{2}^{4}, x_{2}^{-4}\right\}$. Таким образом, каждый автоморфизм графа $\Delta$, стабилизирующий вершину 1 , оставляет на месте каждую из вершин $x_{1}, x_{1}^{-1}$, а потому и каждое из множеств $\left(\Delta\left(x_{1}\right) \cap \Delta(1)\right) \backslash\left\{x_{1}^{-1}\right\}=\left\{x_{1}^{2}, x_{1} x_{2}\right\},\left(\Delta\left(x_{1}^{-1}\right) \cap \Delta(1)\right) \backslash\left\{x_{1}\right\}=\left\{x_{2}, x_{1}^{-2}\right\}$. Итак, с учетом предыдущего каждый автоморфизм графа $\Delta$, стабилизирующий вершину 1 , оставляет на месте следующие множества:

$$
\left\{x_{1}\right\},\left\{x_{1}^{-1}\right\},\left\{x_{2}, x_{1}^{-2}\right\},\left\{x_{1}^{2}, x_{1} x_{2}\right\},\left\{x_{2}^{4}, x_{2}^{-4}\right\},\left\{x_{2}^{-1}, x_{2}^{-1} x_{1}^{-1}\right\} .
$$

Далее, поскольку в графе $\Delta$ имеется не проходящий через 1 путь длины 3 , начинающийся в $x_{2}$ и заканчивающийся в $x_{2}^{4}$, но отсутствует не проходящий через 1 путь длины 3 , начинающийся в $x_{1}^{-2}$ и заканчивающийся в одной из вершин $x_{2}^{4}, x_{2}^{-4}$, то с учетом (2) заключаем, что каждый автоморфизм графа $\Delta$, стабилизирующий вершину 1 , оставляет на месте следующие множества:

$$
\left\{x_{1}\right\},\left\{x_{1}^{-1}\right\},\left\{x_{2}\right\},\left\{x_{1}^{-2}\right\},\left\{x_{1}^{2}, x_{1} x_{2}\right\},\left\{x_{2}^{4}, x_{2}^{-4}\right\},\left\{x_{2}^{-1}, x_{2}^{-1} x_{1}^{-1}\right\} .
$$

Таким образом, для доказательства предложения 6 остается показать, что каждый автоморфизм графа $\Delta$, стабилизирующий вершину 1 , стабилизирует вершины $x_{1}^{2}, x_{1} x_{2}, x_{2}^{-1}, x_{2}^{-1} x_{1}^{-1}$, $x_{2}^{4}, x_{2}^{-4}$.

Поскольку в графе $\Delta$ имеется не проходящий через 1 путь длины 2 , начинающийся в $x_{1}$ и заканчивающийся в $x_{1}^{2}$, но отсутствует не проходящий через 1 путь длины 2 , начинающийся в $x_{1}$ и заканчивающийся в $x_{1} x_{2}$, то с учетом (3) заключаем, что каждый автоморфизм графа $\Delta$, стабилизирующий вершину 1 , оставляет на месте каждую из вершин $x_{1}^{2}, x_{1} x_{2}$. 
Предположим, что имеется такой стабилизирующий вершину 1 автоморфизм $a$ графа $\Delta$, что $a\left(x_{2}^{4}\right)=x_{2}^{-4}$. Согласно (3) при этом $a$ стабилизирует вершину $x_{2}$. Так как в графе $\Delta$ имеется единственный не проходящий через 1 путь длины 3 , начинающийся в $x_{2}$ и заканчивающийся в $x_{2}^{-4}$, а именно путь $\left(x_{2}, x_{2}^{-3}, x_{2}^{-4} x_{1}^{-1}, x_{2}^{-4}\right)$, и имеется не проходящий через 1 путь $\left(x_{2}, x_{2}^{2}, x_{2}^{3}, x_{2}^{4}\right)$ длины 3 , начинающийся в $x_{2}$ и заканчивающийся в $x_{2}^{4}$, то отсюда следует, что $a\left(x_{2}^{2}\right)=x_{2}^{-3}$. Но $x_{2}^{-3} \in \Delta\left(x_{2}^{-4}\right)$, а $a^{-1}\left(x_{2}^{-3}\right)=x_{2}^{2} \notin \Delta\left(x_{2}^{4}\right)=a^{-1}\left(\Delta\left(x_{2}^{-4}\right)\right)$. Полученное противоречие означает, что каждый автоморфизм графа $\Delta$, стабилизирующий вершину 1 , оставляет на месте каждую из вершин $x_{2}^{4}, x_{2}^{-4}$.

Наконец, предположим, что имеется такой стабилизирующий вершину 1 автоморфизм $a$ графа $\Delta$, что $a\left(x_{2}^{-1}\right)=x_{2}^{-1} x_{1}^{-1}$. Как было показано, $а$ стабилизирует вершину $x_{2}^{-4}$. Так как в графе $\Delta$ имеется единственный не проходящий через 1 путь длины 3 , начинающийся в $x_{2}^{-1} x_{1}^{-1}$ и заканчивающийся в $x_{2}^{-4}$, а именно путь $\left(x_{2}^{-1} x_{1}^{-1}, x_{2}^{-1}, x_{2}^{-5}, x_{2}^{-4}\right)$, и имеется не проходящий через 1 путь $\left(x_{2}^{-1}, x_{2}^{-2}, x_{2}^{-3}, x_{2}^{-4}\right)$ длины 3 , начинающийся в $x_{2}^{-1}$ и заканчивающийся в $x_{2}^{-4}$, то отсюда следует, что $a\left(x_{2}^{-2}\right)=x_{2}^{-1}$. Но $x_{2}^{-1} \in \Delta(1)$, а $x_{2}^{-2} \notin \Delta(1)$. Полученное противоречие означает, что каждый автоморфизм графа $\Delta$, стабилизирующий вершину 1 , оставляет на месте каждую из вершин $x_{2}^{-1}, x_{2}^{-1} x_{1}^{-1}$.

Предложение 6 доказано.

3 а м е ч а н и е 2. Указанное в предложении 6 множество $D$ отнюдь не является исключительным. Можно привести примеры других множеств с подобными свойствами.

Предложение 7. Пусть группа $G$ порождается множеством $M=\left\{g_{1}, g_{1}^{-1}, g_{2}, g_{2}^{-1}\right\}$, где $g_{1}^{2} \neq 1 \neq g_{2}^{2}$ и $g_{2} \notin\left\{g_{1}, g_{1}^{-1}\right\}$, причем обхват графа $\Gamma_{G, M}$ больше 20. Положим

$$
M_{1}:=\left\{g_{1}, g_{1}^{-1}, g_{2}, g_{2}^{-1}, g_{1}^{2}, g_{1}^{-2}, g_{1} g_{2}, g_{2}^{-1} g_{1}^{-1}, g_{2}^{4}, g_{2}^{-4}\right\} .
$$

Тогда стабилизатор вершины графа $\Gamma_{G, M_{1}}$ в группе $\operatorname{Aut}\left(\Gamma_{G, M_{1}}\right)$ тривиален (и, следовательно, $\operatorname{Aut}\left(\Gamma_{G, M_{1}}\right)$ совпадает с группой левых сдвигов на элементы из $\left.G\right)$.

Д о к а з а т е л ь с т в о. Пусть $F$ - свободная группа со свободными порождающими $x_{1}, x_{2}$. Каждая вершина $x$ графа Кэли $\Gamma_{F, X}$, где $X=\left\{x_{1}, x_{1}^{-1}, x_{2}, x_{2}^{-1}\right\}$, есть значение однозначно определенного свободно несократимого слова $W_{x}\left(x_{1}, x_{1}^{-1}, x_{2}, x_{2}^{-1}\right)$ над алфавитом $X$. Каждой вершине $x$ шара радиуса 8 с центром 1 графа $\Gamma_{F, X}$ сопоставим вершину $W_{x}\left(g_{1}, g_{1}^{-1}, g_{2}, g_{2}^{-1}\right)$ графа $\Gamma_{G, M}$. В силу условий предложения 7 это отображение индуцирует изоморфизм $\varphi$ подграфа графа $\Gamma_{F, X}$, порожденного шаром радиуса 8 с центром 1 графа $\Gamma_{F, X}$, на подграф графа $\Gamma_{G, M}$, порожденный шаром радиуса 8 с центром 1 графа $\Gamma_{G, M}$, такой, что $\varphi(1)=1$ и ограничение $\varphi$ на шар радиуса 2 с центром 1 графа $\Gamma_{F, D}$, где $D=$ $\left\{x_{1}, x_{1}^{-1}, x_{2}, x_{2}^{-1}, x_{1}^{2}, x_{1}^{-2}, x_{1} x_{2}, x_{2}^{-1} x_{1}^{-1}, x_{2}^{4}, x_{2}^{-4}\right\}$, есть изоморфизм порожденного этим шаром подграфа графа $\Gamma_{F, D}$ на подграф графа $\Gamma_{G, M_{1}}$, порожденный шаром радиуса 2 с центром 1 графа $\Gamma_{G, M_{1}}$. Согласно предложению 6 отсюда следует, что каждый автоморфизм графа $\Gamma_{G, M_{1}}$, стабилизирующий вершину 1 , поэлементно стабилизирует ее окрестность $M_{1}$ в графе $\Gamma_{G, M_{1}}$. Так как для связного вершинно-симметрического графа тривиальность действия стабилизатора вершины в группе всех автоморфизмов графа на окрестности этой вершины влечет тривиальность стабилизатора вершины, то предложение 7 доказано.

3 а м е ч а н и е 3. Имеются также другие весьма общие способы построения связных локально конечных графов Кэли групп с тривиальным стабилизатором вершины в группе всех автоморфизмов графа (см., например, [12] и [11]).

\section{3. Некоторые приложения}

В [8] ставится вопрос о наличии в $\mathfrak{G}$ изолированных бесконечных связных локально конечных вершинно-симметрических унимодулярных графов. Ответ на него содержится в следующей теореме. (Напомним, см. конец разд. 1, что графы Кэли из $\mathfrak{G}$ унимодулярны.) 
Теорема. В $\mathfrak{G}$ имеется изолированный бесконечный граф Кэли (и, следовательно, имеется изолированный бесконечный связный локально конечный вериинно-симметрический граф с унимодулярной группой автоморфизмов).

Д о к а з а т е л ь с т в о. Для доказательства теоремы достаточно указать изолированную бесконечную группу $G$, обладающую такой системой порождающих $M=\left\{g_{1}, g_{1}^{-1}, g_{2}\right.$, $\left.g_{2}^{-1}\right\}$, где $g_{1}^{2} \neq 1 \neq g_{2}^{2}$ и $g_{2} \notin\left\{g_{1}, g_{1}^{-1}\right\}$, что обхват графа Кэли $\Gamma_{G, M}$ больше 20 . Действительно, тогда согласно предложениям 7 и 4 граф Кэли $\Gamma_{G, M_{1}}$, где $M_{1}:=\left\{g_{1}, g_{1}^{-1}, g_{2}, g_{2}^{-1}, g_{1}^{2}, g_{1}^{-2}\right.$, $\left.g_{1} g_{2}, g_{2}^{-1} g_{1}^{-1}, g_{2}^{4}, g_{2}^{-4}\right\}$, обладает требуемым в теореме свойством.

В качестве группы $G$ можно взять, например, группу Р. Томпсона, имеющую обозначение $F$. Действительно, согласно [7, Proposition 5.14] группа $F$ изолирована, а из [5] следует наличие у $F$ такой (явно указываемой в [5]) системы порождающих $M=\left\{g_{1}, g_{1}^{-1}, g_{2}, g_{2}^{-1}\right\}$, где $g_{1}^{2} \neq 1 \neq g_{2}^{2}$ и $g_{2} \notin\left\{g_{1}, g_{1}^{-1}\right\}$, что обхват графа Кэли $\Gamma_{F, M}$ больше 20.

Теорема доказана.

3 а м е ч а н и е 4. Для доказательстве теоремы (точнее, для нахождения такой конечной системы порождающих $X=X^{-1} \not \supset 1$ группы Р. Томпсона $F$, что стабилизатор вершины графа $\Gamma_{F, X}$ в группе $\operatorname{Aut}\left(\Gamma_{F, X}\right)$ тривиален) можно вместо предложения 7 использовать теорему 1.1 из [11] (хорошо известно, что группа $F$ не имеет кручения).

Сходным образом, используя известные результаты, можно показать, что существует неизолированный в $\mathfrak{G}$ граф Кэли (и, следовательно, связный локально конечный вершинносимметрический унимодулярный граф), который не является пределом связных вершинносимметрических конечных графов. Покажем, что таковым является, например, граф Кэли $\Gamma_{G, M_{1}}$ группы Баумслага - Солитера $G=B S(m, n)=\left\langle g_{1}, g_{2}: g_{1} g_{2}^{m} g_{1}^{-1}=g_{2}^{n}\right\rangle$, где целые положительные взаимно простые числа $m, n$ таковы, что $n>m>7$ и $m+n>18$, построенный по системе порождающих $M_{1}:=\left\{g_{1}, g_{1}^{-1}, g_{2}, g_{2}^{-1}, g_{1}^{2}, g_{1}^{-2}, g_{1} g_{2}, g_{2}^{-1} g_{1}^{-1}, g_{2}^{4}, g_{2}^{-4}\right\}$. Хорошо известно, что $G$ - бесконечная неразрешимая группа (причем $g_{1}^{2} \neq 1 \neq g_{2}^{2}$ и $g_{2} \notin\left\{g_{1}, g_{1}^{-1}\right\}$ ). При этом второй коммутант группы $G$ - свободная группа (см. [10]), и следовательно, ряд коммутантов $G^{(k)}, k=0,1,2, \ldots$, группы $G$ (где $G^{(0)}=G$ и $G^{(k)}=\left[G^{(k-1)}, G^{(k-1)}\right]$ при $k>0$ ) строго убывает и имеет единичное пересечение. Кроме того, согласно [13, Proposition 2] обхват графа Кэли $\Gamma_{G, M}$, где $M=\left\{g_{1}, g_{1}^{-1}, g_{2}, g_{2}^{-1}\right\}$, равен $\min \{m+n+2,2 m+6\}>20$. Согласно предложению 7 стабилизатор вершины графа $\Gamma_{G, M_{1}}$ в группе $\operatorname{Aut}\left(\Gamma_{G, M_{1}}\right)$ тривиален, a $\operatorname{Aut}\left(\Gamma_{G, M_{1}}\right)$, следовательно, совпадает с группой левых сдвигов на элементы из $G$.

Для каждого $i \in \mathbb{N}$ пусть $G_{i}:=G / G^{(i)}, M_{1, i}:=\left\{g_{1} G^{(i)}, g_{1}^{-1} G^{(i)}, g_{2} G^{(i)}, g_{2}^{-1} G^{(i)}, g_{1}^{2} G^{(i)}\right.$, $\left.g_{1}^{-2} G^{(i)}, g_{1} g_{2} G^{(i)}, g_{2}^{-1} g_{1}^{-1} G^{(i)}, g_{2}^{4} G^{(i)}, g_{2}^{-4} G^{(i)}\right\}$. Ясно, что последовательность графов Кэли $\left(\Gamma_{G_{i}, M_{1, i}}\right)_{i \in \mathbb{N}}$ сходится к графу Кэли $\Gamma_{G, M_{1}}$. Из тривиальности стабилизатора вершины графа $\Gamma_{G, M_{1}}$ в группе $\operatorname{Aut}\left(\Gamma_{G, M_{1}}\right)$ согласно предложению 1 следует тривиальность стабилизатора вершины графа $\Gamma_{G_{i}, M_{1, i}}$ в группе $\operatorname{Aut}\left(\Gamma_{G_{i}, M_{1, i}}\right)$ (и совпадение $\operatorname{Aut}\left(\Gamma_{G_{i}, M_{1, i}}\right)$ с группой левых сдвигов на элементы из $G_{i}$ ) для всех достаточно больших $i$. С учетом того, что $G_{i} \neq G$ для всех $i \in \mathbb{N}$ (действительно, в отличие от группы $G$ группа $G_{i}$ разрешима), это влечет $\operatorname{Aut}\left(\Gamma_{G_{i}, M_{1, i}}\right) \cong$ $G_{i} \neq G \cong \operatorname{Aut}\left(\Gamma_{G, M_{1}}\right)$ для всех достаточно больших $i$, а потому и $\Gamma_{G_{i}, M_{1, i}} \neq \Gamma_{G, M_{1}}$ для всех достаточно больших $i$. Таким образом, граф $\Gamma_{G, M_{1}}$ является пределом отличных от него связных вершинно-симметрических графов.

С другой стороны, предположим, что граф $\Gamma_{G, M_{1}}$ является пределом последовательности $\left(\Gamma_{i}\right)_{i \in \mathbb{N}}$ связных вершинно-симметрических конечных графов. Тогда, поскольку стабилизатор вершины графа $\Gamma_{G, M_{1}}$ в группе $\operatorname{Aut}\left(\Gamma_{G, M_{1}}\right)$ тривиален и группа $G$ конечно определена, из предложений 1 и 2 следует, что для всех достаточно больших $i$ граф $\Gamma_{i}$ является графом Кэли конечной факторгруппы группы $G$. Но конечная факторгруппа группы $G$ является метациклической (или циклической) группой, а обхват графа $\Gamma_{i}$ для всех достаточно больших $i$ совпадает с обхватом графа $\Gamma_{G, M_{1}}$ и, следовательно, больше 20 . Полученное противоречие (см., например, [6, Theorem 7]) завершает доказательство. 


\section{СПИСОК ЛИТЕРАТУРЫ}

1. Григорчук Р.И. Степени роста конечно-порожденных групп и теория инвариантных средних // Изв. АН СССР. Сер. математическая. 1984. Т. 48, № 5. С. 939-985.

2. Трофимов В.И. Локальное строение графов и полиномиальность роста // Подгрупповое строение групп Свердловск: Изд-во УрО РАН. 1988. С. 149-152.

3. Трофимов В.И. О действии примитивных групп // Алгебра и логика. 1989. Т. 28, № 3. С. 337-363.

4. Трофимов В.И. Группы автоморфизмов графов как топологические группы // Мат. заметки. 1985. Т. 38, вып. 3. С. 378-385.

5. Brin M. The free group of rank 2 is a limit of Thompson's group F// Groups Geom. Dyn. 2010. Vol. 4. P. $433-454$.

6. Condera M., Exoo G., Jajcay R. On the limitations of the use of solvable groups in Cayley graph cage constructions // European J. Combin. 2010. Vol. 31. P. 1819-1828.

7. de Cornulier Y., Guyot L., Pitsch W. On the isolated points in the space of groups // J. Algebra. 2007. Vol. 307. P. 254-277.

8. Frisch J., Tamuz O. Transitive graphs uniquely determined by their local structure // Proc. Amer. Math. Soc. 2016. Vol. 144. P. 1913-1918.

9. Gromov M. Groups of polynomial growth and expanding maps // Inst. Hautes Études Sci. Publ. Math. 1981. Vol. 53. P. 53-73.

10. Kropholler P.H. Baumslag-Solitar groups and some other groups of cohomological dimension two // Comment. Math. Helvetici. 1990. Vol. 65. P. 547-558.

11. Leemann P.-H., de la Salle M. Cayley graphs with few automorphisms.

Available at: Arxiv: 1812.02199v1 [math.CO] 5 Dec 2018.

12. de la Salle M., Tessera R. Characterizing a vertex-transitive graph by a large ball // J. Topology. 2019. Vol. 12. P. 705-743.

13. Stalder Y. Convergence of Baumslag-Solitar groups // Bull. Belg. Math. Soc. Simon Stevin. 2006. Vol. 13, no. 2. P. 221-233.

Трофимов Владимир Иванович

д-р физ.-мат. наук, ведущий науч. сотрудник

Институт математики и механики им. Н.Н. Красовского УрО РАН;

Уральский федеральный университет им. Б.Н. Ельцина

г. Екатеринбург

e-mail: trofimov@imm.uran.ru

Поступила 19.09.2019

После доработки 15.10.2019

Принята к публикации 21.10.2019

\section{REFERENCES}

1. Grigorchuk R.I. Degrees of growth of finitely generated groups, and the theory of invariant means. Math. USSR-Izv., 1985, vol. 25, no. 2, pp. 259-300. doi: 10.1070/IM1985v025n02ABEH001281.

2. Trofimov V.I. The local structure of graphs and the polynomiality of growth. In: Podgruppovoe stroenie grupp (The subgroup structure of groups), Sverdlovsk: Akad. Nauk SSSR Ural. Otdel. Publ., 1988, pp. 149-152 (in Russian).

3. Trofimov V.I. On the action of primitive groups. Algebra and Logic, 1990, vol. 28, no. 3, pp. 220-237. doi: $10.1007 / \mathrm{BF} 01978726$.

4. Trofimov V.I. Automorphism groups of graphs as topological groups. Math. Notes, 1985, vol. 38, no. 3, pp. 717-720. doi: 10.1007/BF01163706 .

5. Brin M. The free group of rank 2 is a limit of Thompson's group F. Groups Geom. Dyn., 2010, vol. 4, no. 3, pp. 433-454. doi: 10.4171/GGD/90 .

6. Condera M., Exoo G., Jajcay R. On the limitations of the use of solvable groups in Cayley graph cage constructions. European J. Combin., 2010, vol. 31, no. 7, pp. 1819-1828. doi: 10.1016/j.ejc.2010.02.002 .

7. de Cornulier Y., Guyot L., Pitsch W. On the isolated points in the space of groups. J. Algebra, 2007, vol. 307, no. 1, pp. 254-277. doi: 10.1016/j.jalgebra.2006.02.012. 
8. Frisch J., Tamuz O. Transitive graphs uniquely determined by their local structure. Proc. Amer. Math. Soc., 2016, vol. 144, no. 5, pp. 1913-1918. doi: 10.1090/proc/12901.

9. Gromov M. Groups of polynomial growth and expanding maps. Publications Mathematiques I.H.E.S., 1981, vol. 53, no. 1, pp. 53-73. doi: 10.1007/BF02698687.

10. Kropholler P.H. Baumslag-Solitar groups and some other groups of cohomological dimension two. Comment. Math. Helvetici, 1990, vol. 65, no. 4, pp. 547-558. doi: 10.1007/BF02566625.

11. Leemann P.-H., de la Salle M. Cayley graphs with few automorphisms. Available at: ArXiv:1812.02199v1 [math.CO] 5 Dec 2018.

12. de la Salle M., Tessera R. Characterizing a vertex-transitive graph by a large ball. Journal of Topology, 2019, vol. 12, no. 3, pp. 705-743. doi: 10.1112/topo.12095.

13. Stalder Y. Convergence of Baumslag-Solitar groups. Bull. Belg. Math. Soc. Simon Stevin, 2006, vol. 13, no. 2, pp. 221-233. doi: 10.36045/bbms/1148059458.

Received September 19, 2019

Revised October 152019

Accepted October 21, 2019

Trofimov Vladimir Ivanovich, Dr. Phys.-Math. Sci., Krasovskii Institute of Mathematics and Mechanics of the Ural Branch of the Russian Academy of Sciences, Yekaterinburg, 620108 Russia; Ural Federal University, Yekaterinburg, 620083 Russia, e-mail: trofimov@imm.uran.ru .

Cite this article as: V. I. Trofimov. On limits of vertex-symmetric graphs and their automorphisms, Trudy Instituta Matematiki i Mekhaniki URO RAN, 2019, vol. 25, no. 4, pp. 226-234. 